Biologisches Institut der Universität Stuttgart

\title{
Wirkungen von Abscisinsäure auf den Kohlenhydrat- und Fettsäurehaushalt von Coleus blumei
}

\author{
Effects of Abscisic Acid on Carbohydrate and Fatty Acid Contents \\ of Coleus blumei
}

U. Kull und M. UNGER

Mit 2 Abbildungen

Eingegangen am 9. Oktober 1973

\section{Summary}

Abscisic acid (ABA) was applied in solutions of $10 \mathrm{ppm}(38,11 \mathrm{M} / \mathrm{I})$ and $100 \mathrm{ppm}(380$ (IM/l) to the apex of young plants of Coleus blumei. The plants showed a weak inhibition of longitudinal growth (significance $\alpha=5 \%$ ).

Application of the phytohormone in leaves and stalks decreased the content of soluble sugars and especially of starch (fig. 1). The fatty acid patterns of saponifiable lipids were investigated quantitatively by gas liquid chromatography. Expressed as \% of total fatry acids the palmitic acid content is raised under the influence of $\mathrm{ABA}$, while the content of linolenic and linoleic acids is diminished (table 1). In \% of dry weight especially the latter two show decreased amounts (fig. 2).

The findings are discussed in relation to the known effects of ABA on stomatal closure, photosynthesis, on changes of permeability of membranes, and in connection with earlier findings on the effects of cytokinins.

\section{Einleitung}

Das in höheren Pflanzen vermutlich ubiquitär verbreitete Phytohormon Abscisinsäure (ABA) hat vorwiegend seneszenzfördernde und wachstumshemmende Wirkungen (AdDicotT und LyON, 1969; Milborrow, 1969). Der Wirkungsmechanismus des Hormons ist weitgehend unklar (ZENK, 1970; Hess, 1973); über den Einfluß auf Speicherungsvorgänge in den Pflanzen ist nur wenig bekannt. Da in der Literatur keine Angaben über eine Wirkung von ABA auf die Kohlenhydratspeicherung zu finden waren und über Effekte auf den Fettsäurehaushalt uns nur die Arbeit von NEwman et al. (1973) bekannt wurde, werden hier einige Befunde vorgelegt. Allerdings sind diese nur an einem Versuchsobjekt gewonnen worden und somit nicht ohne weiteres zu verallgemeinern (vgl. Kull, 1970, 1972). 


\section{Material und Methoden}

\section{Versuchspflanzen}

In Fortsetzung der früheren Untersuchungen über Phytohormonwirkungen auf dic Speicherung wurde Coleus blumet als geeignetes Versuchsobjekt ausgewahlt. An einer anderen Coleus-Art ist von Dorffling und Bottger (1968) gezeigt worden, daß der ABATransport in Internodialsegmenten bei jungem Gewebe vorwiegend basipetal, spater in beiden Richtungen abliuft (vgl. dazu auch Ingersoll und SMITH, 1971). - Die Pflanzen wurden jeweils aus gleichaltrigen Stecklingen eines Klons entnommen und während der Versuchszeit im Gewachshaus bei einer konstanten Temperatur von $20^{\circ} \mathrm{C}$, einer Luftfeuchtigkeıt von $80-90 \%$ und unter natürlichen Lichtverhaltnissen gehalten. Höhe der Pflanzen zu Versuchsbeginn: ca. $8 \mathrm{~cm}$ (2 Blattpaare). Die Versuchszeit war Februar/Marz, so daß KT-Bedingungen herrschten.

\section{ABA-Applikation}

Dic Abscisinsaure (Racemat) wurde als Losung von $10 \mathrm{ppm}(=38, \mu \mathrm{M} / 1$; bezeichnet als A 10) und $100 \mathrm{ppm}$ (= $380 \% \mathrm{M} / \mathrm{l}$; bezeichnet als A 100) mit der Mikropipette auf den Sproßßvegetationskegel aufgebracht, wobei die Pflanzen jeweils $0,01 \mathrm{ml}$ Lösung erhielten. Der Tropfen bleibt dann zwischen den jungen Blattanlagen hängen. Die Kontrollpflanzen erhielten $0,01 \mathrm{ml}$ Aqua dest. Die Applikation erfolgte viermal in wóchentlichem Abstand; eine Woche nach der letzten Behandlung wurde das Material geerntet und durch Gefriertrocknung stabilisiert.

Das Langenwachstum der ABA-behandelten Pflanzen ist schwach gehemmt und beträgt in beiden Versuchsreihen etwa $85 \%$ der Kontrollen; jedoch liegt die Signifikanz nur bei $a$ $=5^{\prime \prime}$ o.

\section{Bestimmung der Inhaltsstoffe}

Die Bestimmung der freien Zucker erfolgte nach früher beschriebenen Methoden (JEREMiAs, 1958, 1965; KuLl., 1965). Die Starkegehalte wurden durch enzymatische Spaltung mit Glucoamylase (Dekkfr u. Richards, 1971) und anschließende Glucosebestimmung mit Glucoseoxidase nach Fleming und Pfglek (1963) gemessen. Für Blattgewebe liefert dieses Verfahren bessere Ergebnisse als die Methode von McCready et al. (1950).

Die Extraktion des Rohfetts, seine Aufarbeitung und die Gaschromatographie der Fettsäuremethylester sind bei KULL und Jerfmias (1972) beschrieben, ebenso die Daten der Gaschromatographie.

\section{Ergebnisse}

\section{Koblenhydratgehalte}

Die Gehalte an Speicherkohlenhydraten in Blättern und Stengeln der ColeusPflanzen sind in Abb. 1 wiedergegeben. Raffinose und Stachyose waren stets nur in Spuren ( $<0,1 \%$ des Trockengewichts) nachweisbar und wurden daher nicht berücksichtigt. Unter dem Einfluß des Hormons beobachtet man eine Mengenabnahme der freien Zucker und der Stärke. Der Effekt ist in den Blättern deutlicher ausgeprägt als in den Stengeln. Glucose ist von den Gehaltsverringerungen relativ am wenigsten betroffen; gut ausgeprägt sind sie bei der Stärke, die das mengenmäßig wichtigste Speicherkohlenhydrat ist. 
Blatter Stengel

$$
1 \% \mathrm{dr}
$$
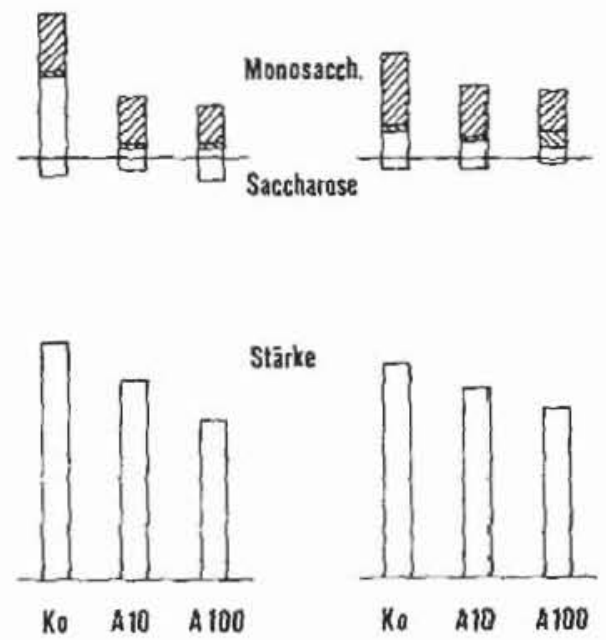

Abb. 1. Verhalten der Kohlenhydrate in Blattern und Stengeln von Colcus blumer bei Behandlung der Pflanzen mit Abscisinsaure. A $10=$ je $0,01 \mathrm{ml}$ ABA-Losung $10 \mathrm{ppm}$, A 100 $=$ je $0,01 \mathrm{ml}$ ABA-Lösung $100 \mathrm{ppm}$ appliziert. Bei den Monosacchariden sind von oben nach unten dargestellt: Glucose, Fructose und Sedoheptulose.

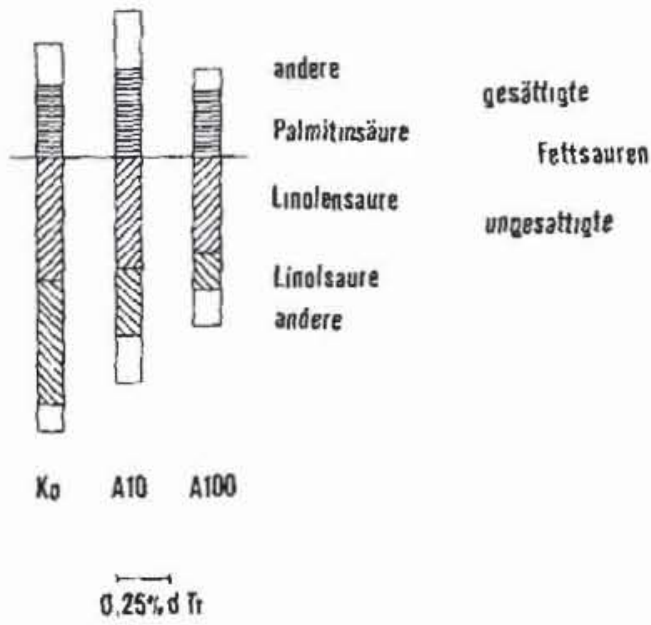

Abb. 2. Verhalten der Fettsäuren (in \% des Trockengewichts) des verseifbaren Anteils des Rohfetts aus Blättern von Coleus blumet bei Behandlung des Pflanzen mit Abscisinsaure. Bezeichnungen A 10 und A 100 wie in Abb. 1.

\section{Fettsäuregehalte}

Der verseifbare Anteil der Rohfett-Fraktion der Coleus-Blätter wurde gaschromatographisch auf die Fettsäurezusammensetzung untersucht. Durch die ABA-Applikation tritt keine qualitative Veränderung ein, jedoch wird der prozentuale Anteil an Palmitinsäure und ungesättigten $\mathrm{C}_{16}$-Säuren erhöht; die Prozentgehalte an Linolen- und Linolsäure sowie an niederen Fettsäuren (bis $\mathrm{C}_{1 s}$ ) nehmen ab (Tabelle 1). 
Tab. 1: Fettsäurezusammensetzung der verseifbaren Lipide (in \% der Gesamtfettsäuren) $\mathrm{Ko}=$ Kontrollen, $\mathrm{A} 10=10 \mathrm{ppm}$ Abscisinsäure, A $100=100 \mathrm{ppm}$ Abscisinsäure.

\begin{tabular}{lrrr}
\hline Fettsaure & Ko & A 10 & A 100 \\
\hline niedermolekulare bis $C_{14}$ & 4,26 & 3,62 & 2,50 \\
$16: 0$ & 18,84 & 25,45 & 28,63 \\
$16: 1+16: 3$ & 1,93 & 3,05 & 7,97 \\
$18: 0$ & 5,81 & 10,01 & 4,78 \\
$18: 1$ & 4,14 & 8,63 & 5,78 \\
$18: 2$ & 19,16 & 18,32 & 16,51 \\
$18: 3$ & 47,12 & 30,56 & 34,26
\end{tabular}

Bezieht man die Fettsäuregehalte auf das Trockengewicht, so ergibt sich das Bild der Abb. 2. Hier wird besonders deutlich, daß vor allem die mehrfach ungesättigten Säuren Linol- und Linolensäure unter ABA-Einfluß abnehmen. Der molare Quotient der Hauptkomponenten Mol Linolensäure + Mol Linolsäure/Mol Palmitinsäure nimmt dementsprechend von 4,32 bei den Kontrollen auf 1,88 bei A 10 und 1,54 bei $A 100 \mathrm{ab}$.

\section{Diskussion}

Die geringe Hemmung des Längenwachstums der Pflanzen steht in Einklang mit Befunden von Tietz (1973) an Erbsenkeimlingen. Die Wirkungen der ABA auf die Kohlenhydratspeicherung sind aufgrund der bekannten physiologischen Wirkungen dieses Hemmstoffes zu erklären. ABA hemmt die Photosynthese intakter und isolierter Blätter (Mittelheuser und van Steveninck, 1971; Poskuta et al., 1972). In der Regel wird dies auf den Verschluß der Stomata durch ABA zurückgeführt (Mittelmeuser und van Steveninck, 1971; Kriedemann et al., 1972). Der Spaltenschluß tritt aber bei Xanthium-Blättern nur an den Applikationsorten ein (Jones und Mansfield, 1972). Wellburn et al. (1973) zeigten, daß die Aktivität der Ribulose-diphosphat-carboxylase in Getreidekeimlingen und isolierten Plastiden durch ABA herabgesetzt wird. ABA hemmt ferner die Chlorophyllsynthese (MERCer und Pughe, 1969) und vermindert den Chlorophyllgehalt (TIETZ, 1972). Eine Herabsetzung der Photosyntheserate erklärt zwanglos die Verringerung der Menge an Speicherkohlenhydraten. Dabei nimmt auch der Stärkegehalt deutlich ab, während ABA-Zufuhr in den Schließzellen der Stomata zu einer Stärkezunahme führt (Mansfield und Jones, 1971). Hinweise auf eine Becinflussung von Invertase durch ABA (SAunders und Poul.son, 1968) ergeben sich aus unseren Daten nicht.

Die Abnahme des Anteils mehrfach ungesättigter Fettsäuren kann in Zusammenhang mit einer Wirkung auf den Plastiden-Stoffwechsel gebracht werden, da die Synthese von Linol- und Linolensäure überwiegend in diesen Organellen stattfindet. Fine generelle Hemmung der Fettsäuresynthese, wie sie bei Ricinus-Keimlingen 
beobachtet wird (ADDicotr und Lyon, 1969), erfolgt in den Coleus-Blättern nicht. Die Mengenabnahme der wichtigen Membranlipid-Bausteine Linolen- und Linolsäure hängt vermutlich mit der Seneszenzförderung zusammen, wie sie an isolierten Blattscheiben durch Colquhoun und Hillman (1972) und Newman et al. (1973) nachgewiesen wurde. Die letztgenannten Autoren finden Veränderungen der prozentualen und der absoluten Gehalte der Fettsäuren, die den unsrigen weitgehend entsprechen; allerdings treten die Effekte bei den isolierten Blattstïcken schon innerhalb von 4 Tagen ein. Außerlich erkennbare Alterungserscheinungen waren an unseren intakten Pflanzen nicht festzustellen.

Veränderungen der Gehalie mehrfach ungesättigter Fettsäuren haben Einfluß auf die Permeabilitätseigenschaften von Membranen. Wirkungen von Abscisinsäure auf Permeabilität und Transportvorgänge sind bekannt (vgl. Glinka und Reinhol.D, 1971; van Steveninck, 1972; Cram und Pitman, 1972). Möglicherweise ist cine Beeinflussung des Membranaufbaus in Plastiden mit eine Ursache des Effektes von ABA auf die Photosynthese (vgl. Weirlburn et al., 1973). Die Zusammensetzung der Membranlipidfraktion kann auch auf die $\Lambda$ ktivität membrangebundener Enzyme des Kohlenhydratstoffwechsels Einfluß nehmen (z. B. ZАKIM et al., 1973, dort weitere Lit.).

Die Veränderungen der Fettsäuregehalte sind denjenigen, die bei Applikation geringer Cytokininmengen (von Kinetin oder Zeatin) eintreten, entgegengesetzt. Hingegen beobachteten wir bei höheren Cytokinin-Gaben gleichartige Effekte (Kul. und Büxenstern, 1974; Kull, unveröffentlicht). Bei der Untersuchung des Wachstums von Sojakallus fanden Blumenfel.D und GazIT (1970) bei Erhöhung der Kinetinkonzentration und konstanter ABA-Menge ebenfalls einen Ubergang von antagonistischer zu synergistischer Wirkung. Versuche, die erkennen lassen sollen, wie rasch die Effekte der ABA auf die Fettsäurezusammensetzung eintreten, sind im Gange.

Für eine Sachbeihilfe haben wir der Deutschen Forschungsgemeinschaft zu danken.

\section{Literatur}

Addicott, F. T., and J. L. Lyon: Ann. Rev. Plant Physiol. 20, 139 (1969).

Blumenfeld, A., and S. Gazit: Plant Physiol. 45, 535 (1970).

Colquhoun, A. J., and J. R. Hillman: Planta (Berl.) 105, 213 (1972).

Cram, W. J., and M. G. Pitman: Austr. J. Biol. Sci. 25, 1125 (1972).

Dekker, R. F. M., and G. N. Richards: J. Sci. Food Agric. 22, 441 (1971).

Dorffling, K., und M. Bóttger: Planta (Berl.) 80, 299 (1968).

Fleming, J. D., and M. F. Pegl.er: Analyst (London) 88, 967 (1963).

Glinka, Z., and L. Reinhold: Plant Physiol. 48, 103 (1971).

Hess, D.: Naturwiss. Rdsch. 26, 284 (1973).

Ingersol., R. B., and O. E. Smith: Plant Cell Physiol. 12, 301 (1971).

Jeremias, K.: Planta (Berl.) 52, 195 (1958).

- Planta (Berl.) 65, 73 (1965).

Jonfs, R. J., and T. A. Mansfield: Physiol. plantarum 26, 321 (1972). 
Kriedemann, P. E., B. R. Loveys, G. L. Fuller, and A. C. Leopold: Plant Physiol. 49, 842 (1972).

- Biochem. Physiol. Pflanzen 161, 330 (1970).

- Bot. Studien (Jena) 19, (1972).

Kult, U.: Beitr. Biol. Pflanzen 41, 231 (1965).

Kull, U., und R Buxenstein: Phytochemistry 13, 39 (1974).

Mansfifl.d, T. A., and R. J. Jones: Planta (Berl.) 101, 147 (1971).

McCready, R. M., J. Guggolz, V. Silvieira, and M. M. Caldwell: Analyt. Chem. 22, 1156 (1950).

Mrrcer, E. I., and J. E. Pughe: Phytochemistry 8, 115 (1969).

Milborrow, B. V.: Sci. Progr. (Oxford) 57, 533 (1969).

Mittel.hflser, C. J., and R. F. M. van Steveninck: Planta (Berl.) 97, 83 (1971).

New.man, D. W., B. W. Rowell, and K. Byrd: Plant Physiol. 51, 229 (1973).

Poskuta, J., R. Antos_e 4 Ski, and M. Faltrynowicz: Photosynthetica 6, 370 (1972).

Saunders, P. F., and R. H. Poulson: in Biochem. and Physiol. of Plant Growth Substances, Ottawa 1968, ed. F. Wightman and G. SFtterfifld, 1581 (1968).

van Steveninck, R. F. M.: Z. Pflanzenphysiol. 67, 282 (1972).

Tietz, A.: Biochem. Physiol. Pflanzen 163, 233 (1972).

- Z. Pflanzenphysiol. 68, 382 (1973).

Welliburn, F. A. M., A. R. Wellburn, J. L. Stoddart, and K. J. Trfharne: Planta (Berl.) 111, 337 (1973).

Zakim, D., J. Goldenberg, and D. A. Vessey: Europ. J. Biochem. 38, 59 (1973).

ZeNK, M H.: Ber. dtsch. bot. Ges. 83, 325 (1970).

U. KulL, Biologisches Institut der Universität Stuttgart D-7coo Stuttgart 60, Ulmer Straße 227. 